\title{
Optimized Angiographic CT Using Intravenous Contrast Injection: A Noninvasive Imaging Option for the Follow-Up of Coiled Aneurysms?
}

\author{
P. Gölitz, T. Struffert, I. Kaschka, K. Roessler, F. Knossalla, and A. Doerfler
}

\begin{abstract}
BACKGROUND AND PURPOSE: Because recanalization of coiled cerebral aneurysms is reported to occur, follow-up imaging is mandatory, ideally noninvasively. Our study aimed to evaluate the accuracy of an optimized angiographic CT by using intravenous contrast material injection in the assessment of coiled cerebral aneurysms, compared with MR angiography and digital subtraction angiography, the criterion standard.
\end{abstract}

MATERIALS AND METHODS: We included 69 patients with 76 coiled cerebral aneurysms. In each patient, we performed an angiographic CT with intravenous contrast material injection with a dual rotational acquisition, a time-of-flight MR angiography, and a DSA. The angiographic CT with intravenous contrast material injection data was postprocessed by using newly implemented reconstructions modes and a dual-volume technique. An aneurysm occlusion rate was assessed in angiographic CT with intravenous contrast material injection and MRA; remnants were measured and correlated with DSA, respectively.

RESULTS: Twenty-eight remnants were revealed by DSA with a mean size of $3.1 \times 3.1 \mathrm{~mm}$. Angiographic CT with intravenous contrast material injection demonstrated a sensitivity of $93 \%$ and a specificity of $96 \%$ in remnant detection. MRA showed almost identical accuracy (sensitivity of $93 \%$, specificity of $100 \%$ ). Assessment of remnant size by angiographic $\mathrm{CT}$ with intravenous contrast material injection and by MRA revealed a high significant correlation with DSA, respectively $(P<.001)$.

CONCLUSIONS: Optimized angiographic CT with intravenous contrast material injection and MRA demonstrated accuracy comparable with that of DSA in the follow-up of coiled aneurysms, respectively. The assessment of remnant size showed a high correlation with DSA for both techniques. Due to the lack of radiation exposure, MRA seems to be the preferred technique. However, angiographic CT with intravenous contrast material injection can be considered a reliable, noninvasive alternative in patients with MR imaging contraindications or in cases of compromising artifacts due to metal implants (ie, clips).

ABBREVIATION: ivACT $=$ angiographic $C T$ with intravenous contrast material injection

$F^{\circ}$ or treatment of cerebral aneurysms, coil embolization has been established as a widely accepted technique. ${ }^{1,2}$ Follow-up evaluation is recommended because recanalization is reported in up to $20 \%$ of aneurysms, ${ }^{3-5}$ with approximately $10 \%$ requiring retreatment. In this instance, DSA is still considered the criterion standard, but it has the disadvantage of being an invasive technique with the risk of procedural complications. ${ }^{6}$ Therefore, ideally, a noninvasive imaging technique is desirable as an alternative to DSA. TOF-MRA and con-

Received January 31, 2014; accepted after revision May 27.

From the Departments of Neuroradiology (P.G., T.S., I.K., A.D.), Neurosurgery (K.R.) and Neurology (F.K.), University of Erlangen, Nuremberg, Germany.

This work was funded by the Medical Valley National Leading Edge Cluster, Erlangen, Germany, Diagnostic Imaging Network, subproject BD 16, research grant No. 13EX1212G, and subproject BD 20, research grant No. 13G 0031A.

Please address correspondence to Philipp Gölitz, MD, Department of Neuroradiology, University of Erlangen-Nuremberg, Schwabachanlage 6, 91054 Erlangen, Germany; e-mail: philipp.goelitz@uk-erlangen.de

http://dx.doi.org/10.3174/ajnr.A4039 trast-enhanced MRA have demonstrated moderate-to-high diagnostic performance. ${ }^{7-9}$ TOF-MRA was superior to contrast-enhanced MRA in terms of coil visibility and is the recommended MR imaging technique. ${ }^{10}$ On the other hand, MR imaging may be impossible due to contraindications or lack of availability.

Here, angiographic CT by using intravenous contrast material injection could be an alternative, noninvasive imaging option. Angiographic CT allows the acquisition of high-resolution data from a rotational run of a C-arm-mounted flat panel detector that differs from conventional CT in the material composing it. Angiographic CT with intravenous contrast material injection (ivACT) has recently demonstrated comparable image quality to DSA in visualizing cerebral artery vasculature ${ }^{11}$ and has been helpful in aneurysm diagnostics ${ }^{12}$ and in the follow-up of clipped aneurysms. ${ }^{13}$ Until now, only angiographic CT with intra-arterial contrast material injection has been investigated in the follow-up of coiled aneurysms, providing promising results. ${ }^{14}$ With the implementation of new reconstruction 
modes and enhanced postprocessing algorithms, the image quality of ivACT could be improved and artifacts could be reduced. Our study aimed to evaluate the accuracy of an optimized, noninvasive ivACT in the follow-up of coiled aneurysms compared with MRA and DSA, the criterion standard.

\section{MATERIALS AND METHODS}

Sixty-nine consecutive patients, 46 women and 23 men with 76 coiled aneurysms, were prospectively enrolled in our study between March 2012 and August 2013 after ethics committee approval and written informed consent were obtained. The average age was $58 \pm 9.3$ years (range, 30-79 years). Forty-three patients had subarachnoid hemorrhage. On average, the aneurysms were $6.0 \pm 3.0 \mathrm{~mm}$ high and $5.4 \pm 3.1 \mathrm{~mm}$ wide, ranging from 2 to 18 $\mathrm{mm}$, respectively. Twenty-five aneurysms originated from the anterior communicating artery, 5 from the MCA, 16 from the tip of the basilar artery, 8 from the posterior communicating artery, 3 from the anterior choroidal artery, 10 from the paraophthalmic segment of the ICA, 5 from the distal carotid bifurcation, 2 from the posterior inferior cerebellar artery, and 2 from the posterior cerebral artery.

In total, 492 coils were used for treatment: 459 bioactive coils loaded with polyglycolic acid (Cerecyte; Codman Neurovascular, Raynham, Massachusetts), 24 standard bare platinum coils (Guglielmi detachable coil; Stryker, Kalamazoo, Michigan), and 9 volume bare platinum coils (Penumbra Coil 400; Penumbra, Alameda, California). Fifteen aneurysms were treated with additional stent protection (Neuroform; Stryker Neurovascular, Fremont, California).

DSA, including a 3D rotational run, was performed in all patients 6 months after treatment. The day after DSA in every patient, TOF-MRA and, thereafter, ivACT was performed after the serum creatinine level was checked.

\section{DSA}

Image acquisition was performed on a biplane flat panel detector angiographic system (Axiom Artis dBA; Siemens, Erlangen, Germany). With standard angiographic methods (transfemoral route), we used a diagnostic catheter for image acquisition. The 3D rotational run was performed with a standard 3D DSA program provided by the manufacturer (5-second DSA run; Siemens). Angiographic data were transferred to a dedicated workstation (Leonardo; Siemens) to generate rotatable, dual-volume images by using commercially available software (iDentify; Siemens). On the basis of the 3D images, targeted 2D series were obtained.

\section{ivACT}

ivACT was performed the day after DSA on the same biplane flat panel detector angiographic system by using a dedicated angiographic CT program (10-second DSA; Siemens), including a native and a contrast-enhanced run. The timing for starting the filling run was done with the "bolus-watching" technique. ${ }^{15}$ First, 60 $\mathrm{mL}$ of contrast material (iomeprol; Imeron 300, Bracco Imaging, Konstanz, Germany) was injected via a needle with a minimal inner diameter of $0.8 \mathrm{~mm}$ into an antecubital vein at a rate of 5 $\mathrm{mL} / \mathrm{s}$ by using a power injector (Accutron HP-D; Medtron, Saarbruecken, Germany) followed by a saline chaser $(60 \mathrm{~mL}$; injection rate, $5 \mathrm{~mL} / \mathrm{s}$ ). Data acquisition per run was performed with the following parameters: acquisition time, 10 seconds per run; 70 $\mathrm{kV} ; 512 \times 512$ matrix; projection on $30 \times 40 \mathrm{~cm}$ flat panel size; $200^{\circ}$ total angle; $0.8^{\circ} /$ frame; 250 frames total; dose, $1.2 \mu \mathrm{Gy} /$ frame; CT weighted dose index, approximately $35 \mathrm{mGy}$. For postprocessing and image evaluation, the data were transferred to a dedicated workstation (Leonardo).

\section{MR Imaging}

MR angiography by using the TOF technique was performed with a 3T scanner (Magnetom Trio; Siemens). The parameters of the TOF-MRA were as follows: TR, $22 \mathrm{~ms}$; TE, $3.98 \mathrm{~ms}$; matrix size, $271 \times 512$; section thickness, $0.7 \mathrm{~mm}$; acquisition time, 5 minutes and 37 seconds. In addition to the source images, MIP reconstructions were generated. For image evaluation, all data were transferred to a dedicated workstation (Leonardo).

\section{Postprocessing and Image Analysis}

Image reconstruction of ivACT and 3D DSA was performed by using the mode "native mask," kernel type "HU," and image impression "sharp" for coil delineation. Next, image reconstruction with the newly implemented, commercially available mode "subtraction with motion correction," kernel type "HU", and image impression "very smooth" was performed for visualization of the arteries. Both datasets were fused and loaded into the InSpace function of the workstation by using a commercially available, dual-volume technique (iDentify) to obtain 3D, freely rotatable images.

The reconstructed and source images of ivACT and MRA were anonymized, stored in random order, and evaluated by 2 experienced neuroradiologists in a consensus reading. Both raters were blinded to the results of the DSA. Evaluation of the DSA images was performed independently by a third experienced neuroradiologist who was not involved in the ivACT and MRA evaluation. For DSA evaluation, the $2 \mathrm{D}$ and the $3 \mathrm{D}$ images, including the source data, were used. For assessment of the occlusion rate, "no remnant" and "any remnant" were differentiated. The category "any remnant" subsumes the categories "neck remnant" and "aneurysm remnant" on the Raymond classification scale. ${ }^{16}$ A more detailed remnant classification was spared to avoid possible bias resulting from discrepancies concerning the correct occlusion grading.

The image quality of ivACT and MRA was assessed by using the following grading system: 2 = no interference; $1=$ compromised, but sufficient image quality; $0=$ not diagnostic.

Statistical analysis was performed by using the software SPSS statistics 20.0 (IBM, Armonk, New York). Mean values and SDs were calculated for aneurysm and remnant size, respectively. The sensitivity and specificity of ivACT and MRA concerning remnant detection were calculated. For analysis of the correlation between the variables, a linear 2-sided correlation (Pearson $r$ ) test was performed. The level of significance was set as $P \leq .05$. Observer agreement with DSA was analyzed for ivACT and MRA by using the $\kappa$ statistic by calculating Cohen $\kappa$ coefficient. The analysis was based on any remnant/no remnant observation per coiled aneurysm. A value of $0.41-0.60$ for the $\kappa$ coefficient was interpreted as moderate agreement; a value of $0.61-0.80$, as substantial agreement; and a value of $0.81-1.00$, as almost perfect agreement. 

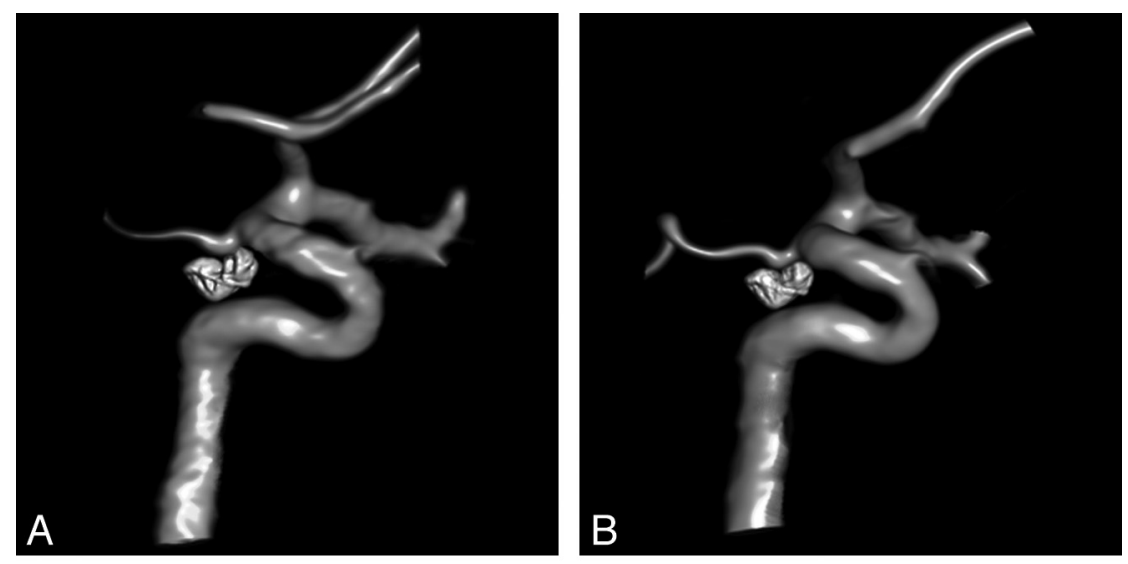

additional aneurysm clip (Fig 5). Image quality was considered compromised by artifacts but sufficient in 4 cases. In the remaining cases, no interfering artifacts were observed.

Remnants were detected correctly in 26 aneurysms, meaning a sensitivity of 93\%. All cases of complete aneurysm occlusion were assessed correctly (specificity of $100 \%)$. Measurements of remnants by MRA demonstrated a significant $(P<$ .001) correlation with DSA (Table). Observer agreement with DSA was found to be $\kappa=0.91(P<.001 ; 95 \% \mathrm{CI}$,

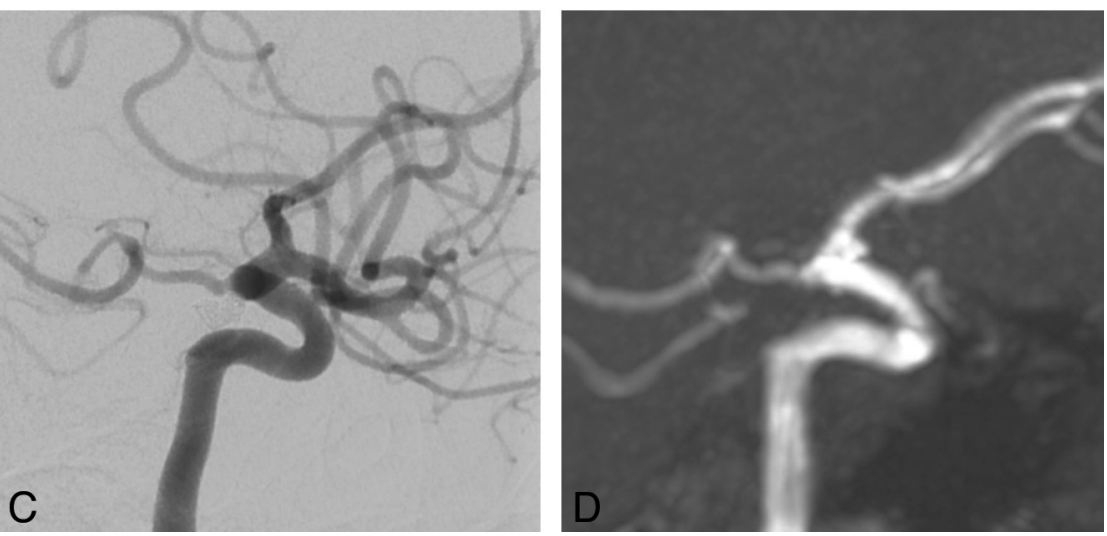
$0.810-1.010)$, indicating almost perfect agreement.

In 1 case of a restless patient, both ivACT and MRA failed in remnant detection because of compromising motion artifacts.

\section{DISCUSSION}

To the best of our knowledge, ivACT has not yet been evaluated for follow-up of coiled aneurysms. In our study, ivACT demonstrated high sensitivity and specificity in detecting remnants down to a minimal size of $1.0 \times 0.9 \mathrm{~mm}$. Assessment of remnant size by ivACT showed a significant correlation with DSA. On the basis of differentiating any remnant and no remnant, agreement of ivACT with DSA

1. ivACT $(A)$ and intra-arterial 3D-DSA $(B)$ show, in comparable image quality, com occlusion after coiling of a posterior communicating artery aneurysm. 2D-DSA (C) confirms complete aneurysm occlusion. TOF-MRA $(D)$ demonstrates complete occlusion.

\section{RESULTS}

Using DSA, we detected a remnant in 28 cases (37\%), and saw complete occlusion in 48 cases $(63 \%)$. On average, aneurysm remnants were $3.1 \pm 1.7 \mathrm{~mm}$ high and $3.1 \pm 2.0 \mathrm{~mm}$ wide, based on DSA imaging. Retreatment was considered necessary in 6 aneurysms, meaning an adequate aneurysm occlusion of $92 \%$.

ivACT could be performed in all patients. In 66 aneurysms, image evaluation was possible without interfering artifacts. Image quality was assessed as compromised but sufficient in 10 aneurysms. No case was assessed as nondiagnostic.

By ivACT, complete aneurysm occlusion was assessed correctly in 46 aneurysms, meaning a specificity of 96\% (Figs 1 and 2). In 1 of the 2 cases in which remnants were supposed incorrectly, volume bare platinum coils were used, inducing compromising beam-hardening artifacts. In 26 aneurysms, remnants were detected correctly, meaning a sensitivity of 93\% (Fig 3). In the 2 cases in which ivACT failed, only small neck remnants without indication for retreatment were missed. Overall, remnants up to a minimal size of $1.0 \times 0.9 \mathrm{~mm}$ could be revealed by ivACT. Implanted stents caused no substantial artifacts (Fig 4). Measurement of remnants by ivACT demonstrated a significant $(P<.001)$ correlation with DSA (Table). Observer agreement with DSA was $\kappa=0.89(P<.001 ; 95 \% \mathrm{CI}, 0.779-0.995)$, indicating almost perfect agreement.

MRA was performed in 67 patients. In 2 patients, MRA was not viable because of a cardiac pacemaker. In 1 case, MRA was assessed as nondiagnostic due to susceptibility artifacts from an was identical in assessing the aneurysmal occlusion rate.

ivACT was performed as a dual rotational 10-second acquisition run by using the bolus-watching technique to obtain the best arterial vessel opacification. Using a dual-volume technique (iDentify) with newly implemented reconstruction modes, one can generate freely rotatable 3D images. Fusing 2 separately reconstructed datasets, 1 optimized for vessel visualization and 1 for coil delineation, allows an improvement in image quality compared with MPR images that are obtained from only 1 reconstruction step.

An advantage compared with DSA is that ivACT allows evaluation of several coiled aneurysms in only 1 examination step (comparison shown in Fig 2). Implanted stents after stent-assisted coiling induced no substantial artifacts (comparison shown in Fig 4). Moreover, the stent lumen can be assessed precisely in ivACT, ${ }^{17}$ which is not achievable in MRA. In the case of volume bare platinum coils, ivACT might be less suitable than MRA because these coils seem to induce compromising beam-hardening artifacts. However, our results concerning these coils are limited due to the small sample size, but that might be a subject for further investigations.

In angiographic CT using intra-arterial contrast material injection, substantial "eggshell" artifacts are reported for coil packages larger than $10 \mathrm{~mm}$ in diameter, supposedly the result of insufficient reconstruction algorithms. ${ }^{12}$ In contrast, in our study by using newly implemented reconstruction algorithms and 

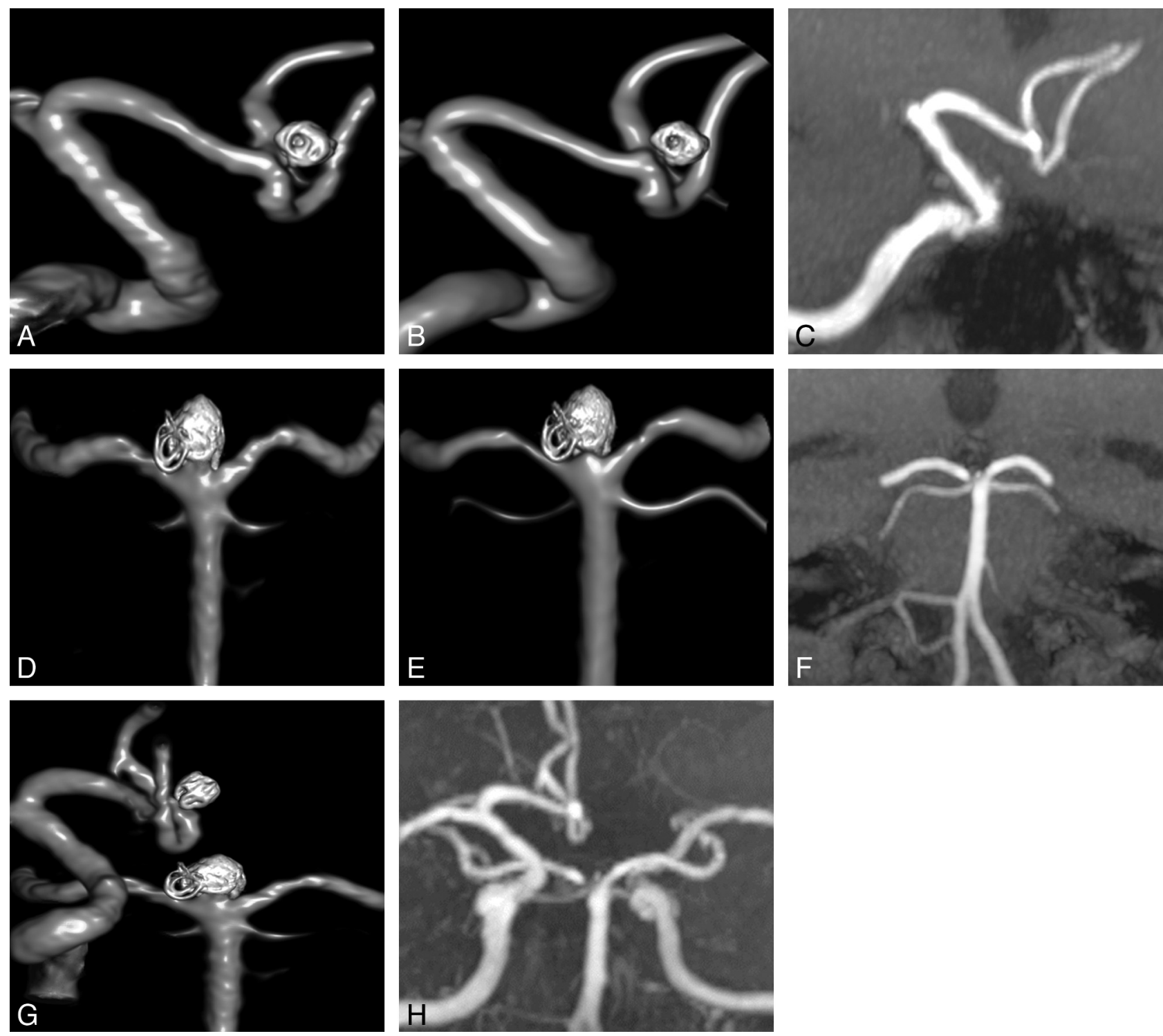

FIG 2. ivACT $(A)$ and intra-arterial 3D-DSA $(B)$ show, in a comparable way, complete occlusion of an anterior communicating artery aneurysm after coiling. MRA reveals no remnant $(C)$. In addition, a second aneurysm located at the tip of the basilar artery was treated by coil embolization. $\operatorname{ivACT}(D)$ and 3D-DSA (E) demonstrate, in nearly identical quality, complete aneurysm occlusion with only mild irregularity at the aneurysm base. In addition, MRA reveals no remnant $(F)$. As an advantage of ivACT $(G)$ and MRA $(H)$, aneurysm evaluation can be performed with only 1 examination step, in contrast to DSA, requiring several vessel catheterizations and contrast material injections.

iDentify instead of MPR reconstructions, we did not observe such artifacts in larger aneurysms. Additionally, image evaluation might be facilitated by injecting the contrast material intravenously, leading to a homogeneous contrast mixing without highflow artifacts, which can occur with intra-arterial injection.

TOF-MRA also demonstrated high sensitivity and specificity in remnant detection on almost the same level in ivACT. Assessment of remnant size was comparable with that of ivACT and demonstrated a significant correlation with DSA. Stents induced no artifacts substantially compromising aneurysm evaluation, but a reliable assessment of the stent lumen is not viable in MRA.

Our results are in line with those in other MR imaging studies that found substantial agreement between DSA and MRA in evaluating coiled aneurysms. The reported sensitivity and specificity of remnant detection are comparable with our results. ${ }^{8,18,19}$ One study found MRA sufficient for therapeutic decision-making in the retreatment of recanalization. ${ }^{18}$ On the other hand, evaluation of MRA performed with 3T MR imaging revealed a greater discrepancy with DSA in aneurysms with a small (1-3 $\mathrm{mm})$ residual lumen than in those with a larger lumen because for a small residual lumen, the precise differentiation between subtotal and incomplete occlusion does not seem possible. ${ }^{8}$ Similarly, sufficient accuracy of MRA is reported, in general, for the screening of residual flow after coil embolization, but in particular in small aneurysms $(<6 \mathrm{~mm})$, the detection of residual flow was limited. ${ }^{17}$ Therefore, ivACT might be considered more favorable than MRA in small aneurysms because we found no restrictions with ivACT in evaluating small aneurysms. On the contrary, because ivACT might be limited in evaluating large aneurysms with a maximum diameter of $>10 \mathrm{~mm}$, ivACT and MRA could be considered complementary imaging techniques, which might be selected individ- 

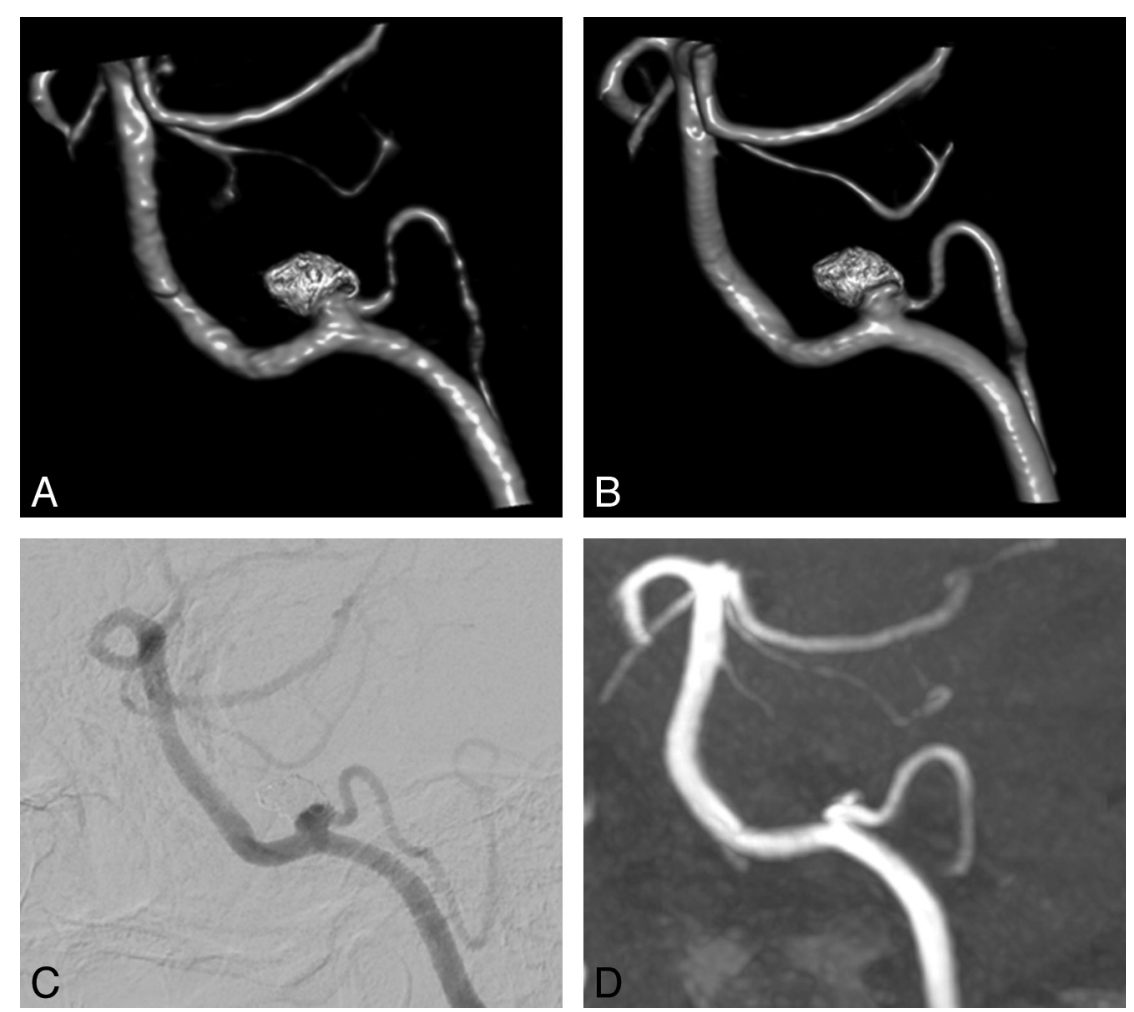

FIG 3. ivACT $(A)$ and $3 D-D S A(B)$ reveal a small remnant after coiling of a posterior inferior cerebellar artery aneurysm in nearly identical quality. DSA confirms this finding (C). TOF-MRA also detects the small remnant $(D)$.
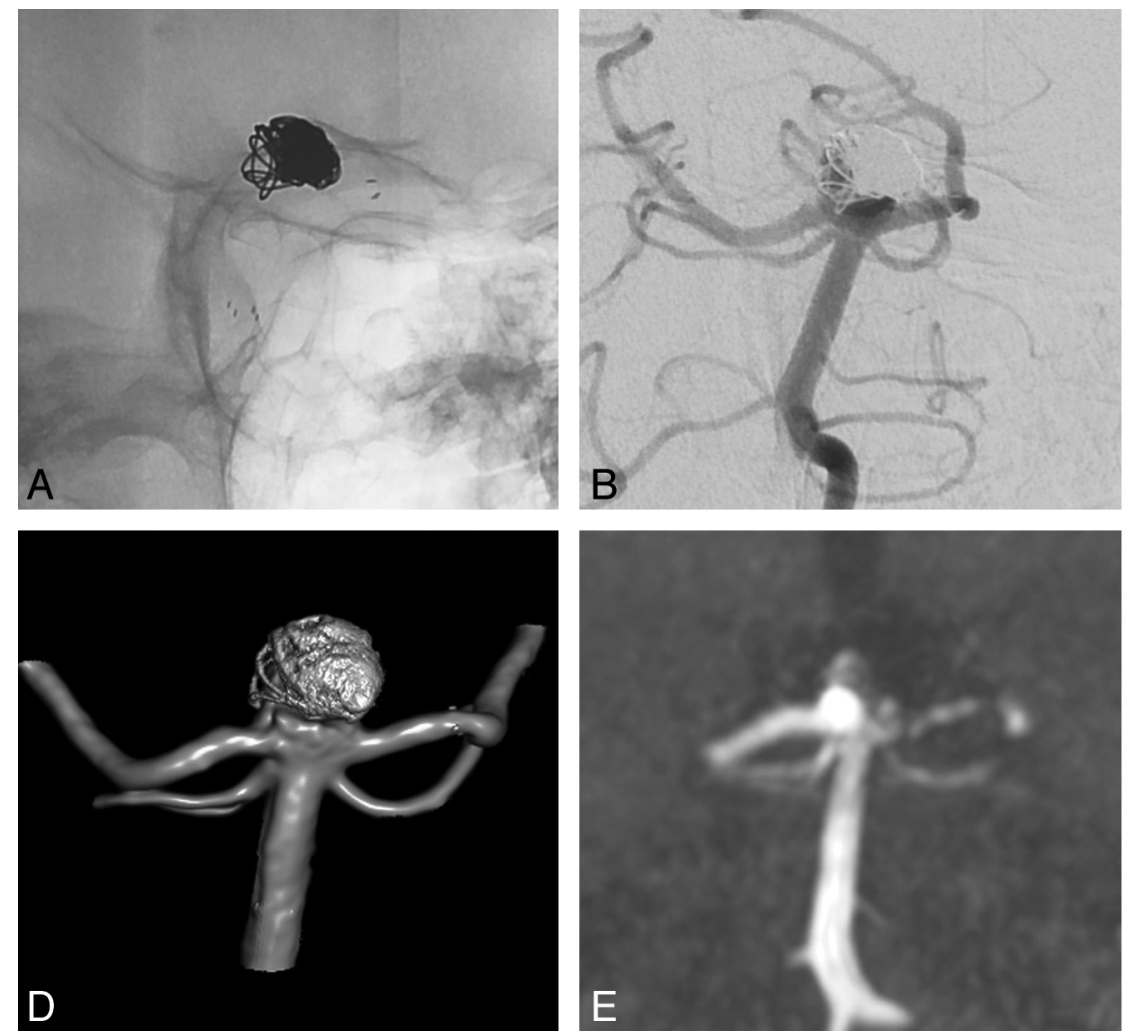

ually, depending on aneurysm size or coil material. Here, further investigations with an increased patient population are necessary.

In our study, observer agreement with DSA was almost perfect for both ivACT and MRA. Interobserver variability was not assessed because our study aimed first to demonstrate the feasibility of ivACT and to assess ivACT and MRA as close as possible to the usual clinical setting with only 1 reader. Because MRA and ivACT reached nearly identical accuracy in remnant detection and in the assessment of remnant size, they seem equally capable of a noninvasive follow-up; both may be reliable alternatives to DSA. However, because MRA can be performed without radiation exposure to the patient, it may serve as the preferred technique in routine cases. Nevertheless, in patients with contraindications to MR imaging (heart pacemaker and so forth) or in the case of compromising susceptibility artifacts due to additional metal implants (eg, aneurysm clips, comparison shown in Fig 5), ivACT can be considered a reliable alternative. ivACT could also be an option in

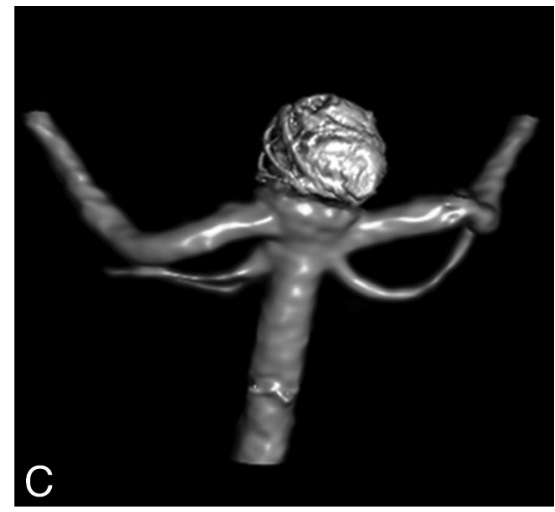

C

FIG 4. Native $(A)$ and DSA images $(B)$ after stent-assisted coil embolization of a basilar artery tip aneurysm show a small, laterally located remnant. In ivACT (C) and intra-arterial rotational 3D-DSA (D), the remnant can be revealed reliably in nearly identical image quality. In TOF-MRA $(E)$, the remnant can be delineated clearly, but in contrast to ivACT and 3D-DSA, artifacts are induced by the implanted stent, making stent assessment impossible. In contrast, ivACT allows precise assessment of the stent lumen. 
Mean size and SD of aneurysm remnants measured by ivACT and TOF-MRA in correlation with DSA

\begin{tabular}{lccccc}
\hline Remnant & $\begin{array}{c}\text { DSA } \\
\text { (Mean) (SD) }\end{array}$ & $\begin{array}{c}\text { ivACT } \\
\text { (Mean) (SD) }\end{array}$ & $\begin{array}{c}\text { Pearson } \\
\text { Correlation Coefficient }\end{array}$ & $\begin{array}{c}\text { TOF-MRA } \\
\text { (Mean) (SD) }\end{array}$ & $\begin{array}{c}\text { Pearson } \\
\text { Correlation Coefficient }\end{array}$ \\
\hline Height & $3.1 \mathrm{~mm}( \pm 1.7)$ & $2.9 \mathrm{~mm}( \pm 1.8)$ & $0.963(P<.001)$ & $2.8 \mathrm{~mm}( \pm 1.9)$ & $0.925(P<.001)$ \\
Width & $3.1 \mathrm{~mm}( \pm 2.0)$ & $3.1 \mathrm{~mm}( \pm 2.6)$ & $0.930(P<0.001)$ & $2.8 \mathrm{~mm}( \pm 2.1)$ & $0.941(P<.001)$ \\
\hline
\end{tabular}
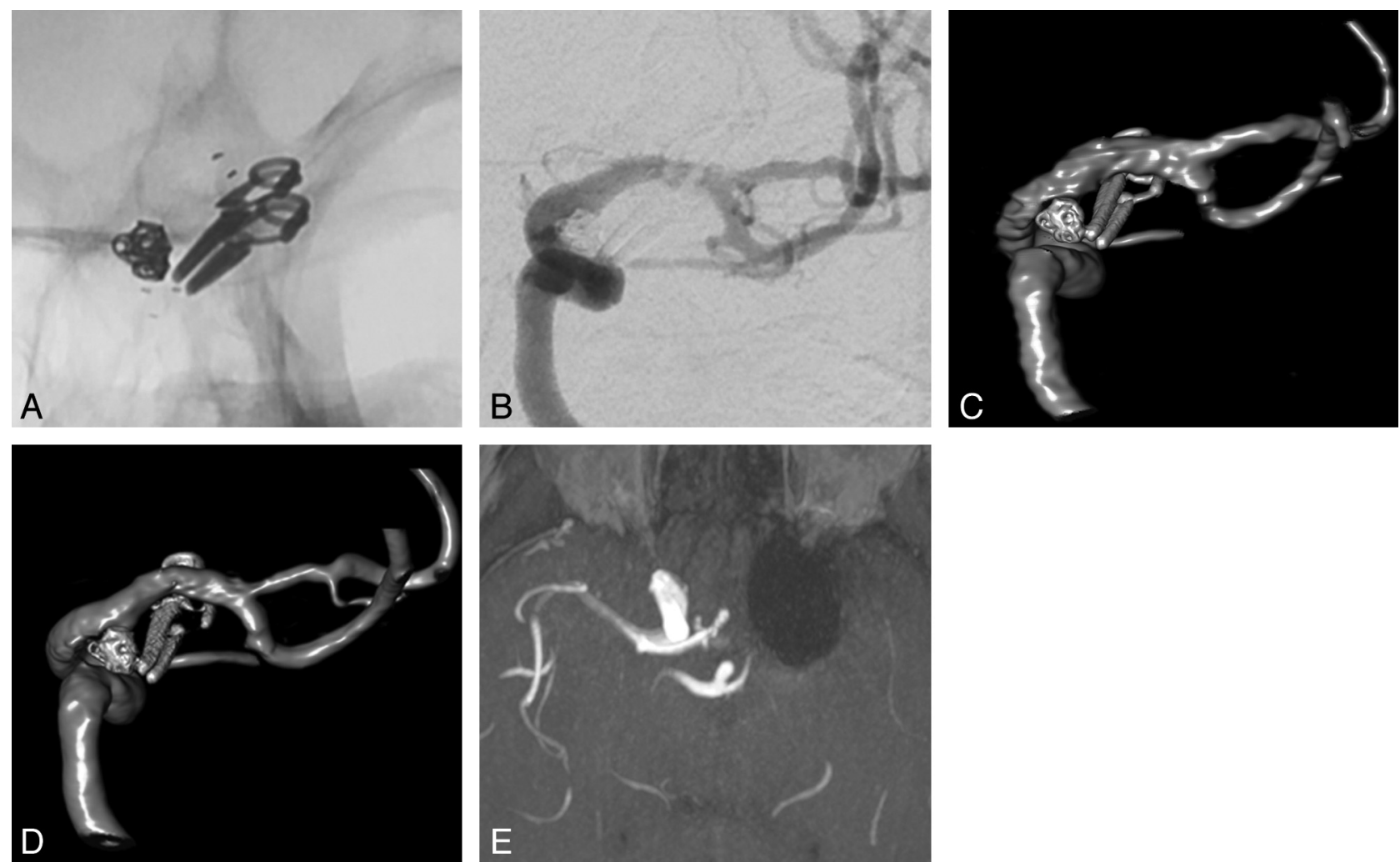

FIG 5. Native $(A)$ and contrast-enhanced 2D-DSA $(B)$ images show complete occlusion of a posterior communicating artery aneurysm, treated initially by subtotal clipping, followed by stent-assisted coil embolization. ivACT (C) and 3D-DSA (D) illustrate complete aneurysm occlusion in a comparable quality. In ivACT, no compromising artifacts by the metal implants are observed. MRA fails in delineating the coiled aneurysm because of severe susceptibility artifacts $(E)$.

patients treated with ventriculopertioneal shunts to avoid potential maladjustment or damage. The advantages of ivACT are that it has a short acquisition time, it is less time-consuming than DSA and MRA, and it can be performed on an outpatient basis, of interest from an economic point of view.

Concerning radiation exposure, evaluations by using an Alderson-Rando phantom revealed an effective dose of $2.9 \mathrm{mSv}$ for the 10-second run of an ivACT if the entire head was scanned. However, by collimating the FOV on the coil package, the radiation dose can be substantially reduced by up to $0.5 \mathrm{mSv} .^{20}$ For comparison, the effective dose of a biplane 2D-DSA series was measured as $1 \mathrm{mSv}$. Thus, especially in the follow-up of multiple coiled cerebral aneurysms, the use of ivACT could significantly reduce radiation exposure to the patient compared with DSA. Advances in the rapidly developing flat panel detector technology might further contribute to increasing dose effectiveness and improving image quality.

Our findings are limited because mainly 1 type of coil was used for aneurysm treatment in our study population. Moreover, the mean aneurysm size and the mean remnant size were relatively low in our population; thus, an objective for further investigations could be to add more large aneurysms to our series. Head motion and the resulting artifacts lead to a compromised image quality in both modalities, making restless or noncompliant patients less suitable for both imaging techniques. The development of special cups for better head fixation might be helpful for reducing motion artifacts in ivACT. As an objective for further investigations, the implementation of reconstruction modes with automated metal artifact reduction might further contribute to improved ivACT image quality.

\section{CONCLUSIONS}

Both, ivACT and MRA, have a high accuracy in detecting remnants after coiling. The accuracy of ivACT as well as of MRA is comparable with the accuracy of DSA. Both noninvasive modalities allowed a reliable assessment of the aneurysm occlusion rate and remnant size. Due to the lack of radiation exposure, MRA seems to be the preferred imaging technique. However, in selected patients, ivACT can be considered an alternative (ie, in patients with contraindications to MR imaging or in the case of compromising artifacts due to metal implants such as aneurysm clips). Moreover, MRA and ivACT might also be considered complementary imaging techniques, which could be selected depending on aneurysm size or coil material. Further investigations in a 
larger patient population are needed to substantiate our initial results.

\section{REFERENCES}

1. Pierot L, Spelle L, Vitry F, et al. Immediate clinical outcome of patients harboring unruptured intracranial aneurysms treated by endovascular approach: results of the ATENA study. Stroke 2008;39:2497-504

2. Lanzino G, Murad MH, d'Urso PI, et al. Coil embolization versus clipping for ruptured intracranial aneurysms: a meta-analysis of prospective controlled published studies. AJNR Am J Neuroradiol 2013:34:1764-68

3. Ferns SP, Sprengers ME, von Rooij WJ, et al. Coiling of intracranial aneurysms: a systematic review on initial occlusion and reopening and retreatment rates. Stroke 2009;40:e523-29

4. Campi A, Ramzi N, Molyneux AJ, et al. Retreatment of ruptured cerebral aneurysms in patients randomized by coiling or clipping in the International Subarachnoid Aneurysm Trial (ISAT). Stroke 2007;38:1538-44

5. Pierot L, Delcourt C, Bouquigny F, et al. Follow-up of intracranial aneurysms selectively treated with coils: prospective evaluation of contrast-enhanced MR angiography. AJNR Am J Neuroradiol 2006;27:744-49

6. Fifi JT, Meyers PM, Lavine SD, et al. Complications of modern diagnostic cerebral angiography in an academic medical center. $J$ Vasc Interv Radiol 2009;20:442-47

7. Anzalone N, Scomazzoni F, Cirillo M, et al. Follow-up of coiled aneurysms at 3T: comparison of 3D time-of-flight MR angiography and contrast-enhanced MR angiography. AJNR Am J Neuroradiol 2008;29:1530-36

8. Schaafsma JD, Velthuis BK, Majoie CB, et al. Intracranial aneurysms treated with coil placement: test characteristics of follow-up MR angiography—multicenter study. Radiology 2010;256:209-18

9. Cho WS, Kim SS, Lee SJ, et al. The effectiveness of 3T time-of-flight magnetic resonance angiography for follow-up evaluations after the stent-assisted coil embolization of cerebral aneurysms. Acta Radiol 2013;55:604-13

10. Pierot L, Portefaix C, Gauvrit JY, et al. Follow-up of coiled intracra- nial aneurysms: comparison of 3D time-of-flight MR angiography at $3 \mathrm{~T}$ and $1.5 \mathrm{~T}$ in a large prospective series. AJNR Am J Neuroradiol 2012;33:2162-66

11. Saake M, Breuer L, Goelitz P, et al. Flat detector computed tomography angiography with intravenous contrast application: feasibility for visualization of cerebral arterial vasculature. J Neuroimaging 2013;23:414-20

12. Gölitz P, Struffert T, Knossalla F, et al. Angiographic CT with intravenous contrast injection compared with conventional rotational angiography in the diagnostic work-up of cerebral aneurysms. AJNR Am J Neuroradiol 2012;33:982-87

13. Gölitz P, Struffert T, Ganslandt O, et al. Optimized angiographic computed tomography with intravenous contrast injection: an alternative to conventional angiography in the follow-up of clipped aneurysms? J Neurosurg 2012;117:29-36

14. Buhk JH, Kallenberg K, Mohr A, et al. Evaluation of angiographic computed tomography in the follow-up after endovascular treatment of cerebral aneurysms: a comparative study with DSA and TOF-MRA. Eur Radiol 2009;19:430-36

15. Struffert T, Kloska S, Engelhorn T, et al. Optimized intravenous flat detector CT for non-invasive visualization of intracranial stents: first results. Eur Radiol 2011;21:411-18

16. Roy D, Milot G, Raymond J. Endovascular treatment of unruptured aneurysms. Stroke 2001;32:1998-2004

17. Struffert T, Ott S, Adamek E, et al. Flat-detector computed tomography in the assessment of intracranial stents: comparison with multi detector CT and conventional angiography in a new animal model. Eur Radiol 2011;21:1779-87

18. Schaafsma JD, Velthuis BK, van den Berg R, et al. Coil-treated aneurysms: decision making regarding additional treatment based on findings of MR angiography and intraarterial DSA. Radiology 2012;265:858-63

19. Lavoie P, Gariépy JL, Milot G, et al. Residual flow after cerebral aneurysm coil occlusion: diagnostic accuracy of MR angiography. Stroke 2012;43:740-46

20. Struffert T, Hauer M, Banckwitz R, et al. Effective dose to patient measurements in flat-detector and multislice computed tomography: a comparison of applications in neuroradiology. Eur Radiol 2014;24: 1257-65 